\title{
Two Dimensional Flow Model for Analysis of Expiratory Check Valve
}

\author{
Tamotsu Takishima, Hidetada Sasaki and Takashi \\ NAKamuRA \\ The First Department of Internal Medicine,* Tohoku \\ University School of Medicine, Sendai
}

Takismima, T., Sasaki, H. and Nakamura, T. Two Dimensional Flow Model for Analysis of Expiratory Check Valve. Tohoku J. exp. Med., 1972, 106 (4), 311 327 We made a two-dimensional-flow model composed of two bands in parallel, the roof of which was made up with the condome rubber collapsible and segmented into five parts to keep interdependence in movement of each segment and the bottom with the plastic plate. In this model, pressure flow relationships demonstrated the flow to reach its maximum at a certain driving pressure, and the lower the compliance of the elastic roof, the higher the maximum flow rate established. Displacement of the collapsible roof toward the bottom occurred just at a very limited segment near the outlet of the airway. Combining the theoretical analysis with our experimental findings on dogs, it is concluded that the maximum flow has an inverse relation with both compliance and resistance of the bronchi. The fact that displacement of the collapsible roof toward the bottom appears at very limited segment, means stress on the membrane to focus into much narrower segment and might indicate "expiratory check valve" to occur also at a very limited segment of bronchi. lung; airway; bronchi; flow model; compliance; expiratory check valve

Decrease of the maximum expiratory flow of the tracheobronchial tree, that is "expiratory check valve" which markedly limits the life activity of patients with chronic obstructive lung disease, may be caused by the following two major physiological mechanisms: (a) an increase of transmural pressure and (b) the collapsibility of the tree. Airway obstruction may diminish the maximum expiratory flow by augmenting transmural pressure of the airway, while destruction of the lung parenchyma and the bronchi supposedly diminishes the flow by increasing collapsibility of the airway.

Fry (1958) presented an elegant mathematical analysis on a rather simple model composed of a collapsible tubing and an elastic balloon in series, suggesting that the maximum expiratory flow was a function of both compliance and resistance of the tubing. On the other hand, Mead and associates (1967) defined the maximal flow by relationship between the elastic recoil pressure and the upstream resistance according to their equal pressure point (EPP) concept. They

Received for publication, September 7, 1971.

* Director: Prof. T. Nakamura. 
say that under conditions of maximal flow EPP divides the airways into upstream segments of fixed geometry, and once the maximal flow has been achieved, one can think that it is determined by lung recoil and the resistance of the upstream segment. As a result, in their equation the compliance factor of the airway is dropped as the determinant of expiratory flow. Pride and co-workers (1967) made another approach and suggested the collapse properties or smooth muscle tone of airways as an important factor to determine the maximal expiratory flow.

Although these authors have thought that increased compressibility should have an influence on the maximum flow, they have not made any attempt directly to explain the exact mechanisms by which the flow is limited; that is, to what extent does the collapsibility of bronchi limit the expiratory flow or how is the maximum flow established?. Even in the analysis reported by Fry et al., they have defined the tube as having a constant value of compliance, which cannot tell us any information about how the collapse along the airway occurs as the maximum flow is reached.

In the present study we have evaluated how the collapse along the airway occurs and to what extent the collapsibility associates with a limitation of expiratory flow, using the two-dimensional flow model composed of parallel collapsible bands, through which the flow can occur.

\section{EXPERIMENT}

To test the expiratory check valve, many investigators have used a piece of elastic tubing, e.g., Penrose rubber (Fry 1958). The tubing model, however, has a number of definite disadvantage as follows. First, the tubing can collapse but in a quite complex way, making it difficult to observe airway narrowing quantitatively. Secondly, the tubing has rather continuous structure, a part of which hardly makes any deformation independently from others. In other words, narrowing of the tubing at a limited portion, if any, is necessarily accompanied with a change in shape of the neighboring segment. This behavior is not in favor of simulating stress-strain characteristics of the bronchus which usually shows much less longitudinal interdependence than the homogeneous rubber tubing.

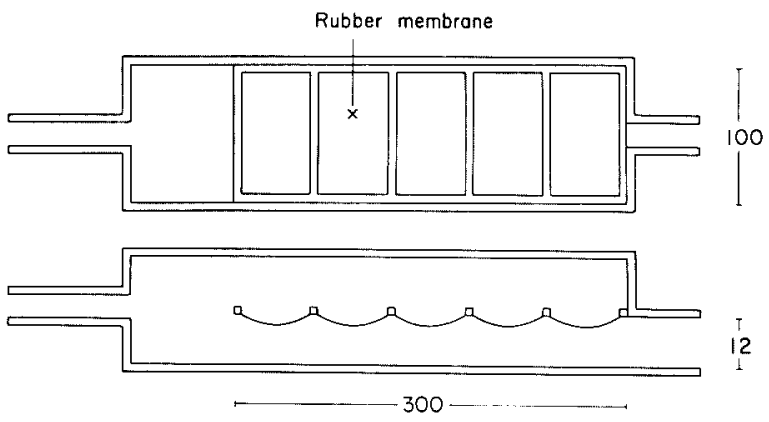

Fig. 1. Diagram of the present flow-model. For explanation, see text. 
To solve the problems mentioned above, we made the quasi-two-dimensional flow-model composed of two collapsible bands in parallel instead of the tubing (Fig. 1). The roof of the parallel bands is made up with the collapsible condome rubber and the bottom with the plastic plate. To keep interdependence in movement of different parts of the membrane as possible, latex rubber was divided into five parts by thin rigid ribs which could not displace. Flow can occur through the channel when it is positioned in the box which can generate a driving pressure, and the driving pressure in the box can also compress the elastic roof toward the bottom, resulting in an increase of airway resistance.

\section{1) Pressure-flow relationship in the parallel bands}

Before observing behavior of the collapsible bands, pressure-flow relations in the parallel bands must be obtained.

Assume a laminar flow to the direction of the length through the parallel band which is $m \mathrm{~cm}$ of width, $l \mathrm{~cm}$ of length and $H \mathrm{~cm}$ of height between two bands, provided the width of the band is much larger than the height so that lateral walls of the system do not influence the flow. In the channel, a profile of the flow

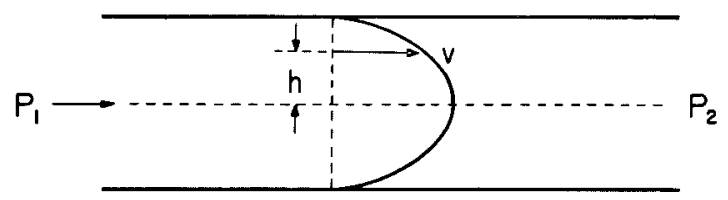

Fig. 2. Profile of the flow in the model. v, linear velocity of the flow; h, height from the middle of the stream; and $P_{1}, P_{2}$, applied pressures.

shows parabolic shape to the direction of the flow, linear velocity of which is zero at the surface of the bands and is maximum at the middle of the space, as shown in Fig. 2.

$P t$ is defined as the sheer stress per unit area at height $h$ from the middle of the stream and $P$ as applied pressure between the inlet and the outlet of the channel to the direction of the length. Then, the total force applied to the gas $l \mathrm{~cm}$ of length and $2 h \mathrm{~cm}$ of height will be $2 h m \times \Delta P$, and the total sheer stress produced at the total lateral surface of the gas will be $2 \mathrm{~lm} \times P t$. At the steady state the summed applied pressure must be counterbalanced with summed sheer stress as follows:

$$
2 h m \Delta P=2 \operatorname{lm} P t
$$

Therefore,

$$
P t=\frac{h}{l} \Delta P
$$

There exists a gradient of flow velocity in the space, showing a parabolic distribution. Defining $v$ as flow velocity at height $h$ from the middle of the space, 
sheer stress at the surface must vary in direct proportion to velocity gradient of the flow as follows:

$$
P t=-\frac{d v}{d h}
$$

where $\eta$ is viscosity coefficient of gas, and from equation (1) and (2)

$$
d v=-\frac{P t}{\eta} d h=-\frac{h \Delta P}{\eta l} d h
$$

The integral of eq. (3) gives the next equations.

$$
\int d v=-\frac{P}{\eta l} \int h d h
$$

and

$$
v=\frac{P}{2 \eta l}\left(\frac{H^{2}}{4}-h^{2}\right)
$$

Eq. (4) shows that velocity profile of the flow in parallel bands appears parabolic with velocity maximum at the middle of the space.

From eq. (4) volume flow can be calculated as follows;

$$
V=2 \int_{0}^{H / 2} m v d h=\frac{m H^{3} \Delta P}{12 \eta l}
$$

Eq. (5) is similar to the equation of Hagen-Poiseuille which is obtained from flow-pressure relation in the cylindrical tubing, except the power of the height $H$ being third instead of fourth.

We made plastic bands in parallel with the walls at both sides $100 \mathrm{~mm}$ of width $(m), 300 \mathrm{~mm}$ of length $(l)$, and $1,1.65,2$ and $3 \mathrm{~mm}$ of height $(H)$ to testify our theoretical calculation, and observed pressure-flow relation in the system. Volume flow obtained by a pneumotachometer of the Fleisch type attached to the inlet of the channel and pressure difference between two sides of the system by the strain gauge electromanometer.

Fig. 3 shows laminar coefficient of the resistances obtained at different heights of the bands. The regression line drawn by eyes indicates laminar coefficient being proportional to 2.8 power of the height $(H)$, which roughly coincides with our theoretical consideration. The discrepancy between the experimental results obtained and the theory could be explained by several factors including turbulence in the flow, characteristics of the plastic plate and other complicating aerodynamies. For simplicity, in the following consideration we use the theoretical data rather than that actually obtained.

\section{2) Behavior in the mathematical model}

Let us consider a mathematical model of the parallel bands for height $H$ as in Fig. 4, of which the bottom is made up with plastic band and the roof with collap- 


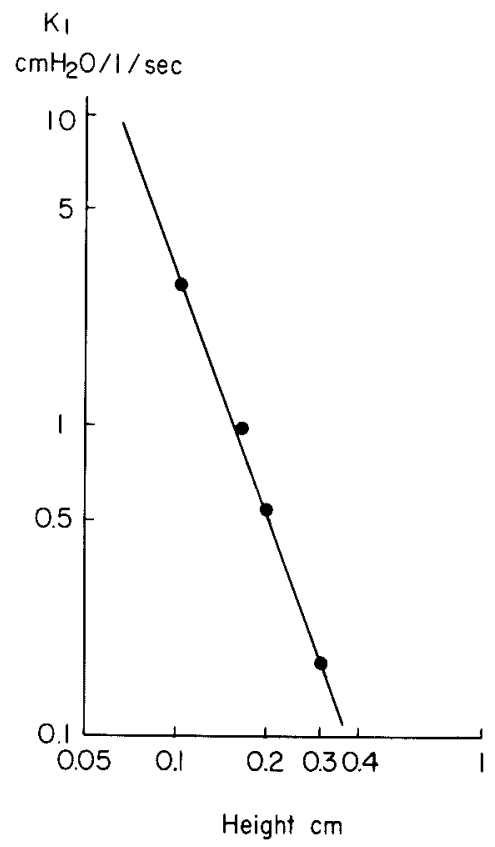

Fig. 3. Laminar coefficient in the resistance of the flow channel with different height between two parallel bands.

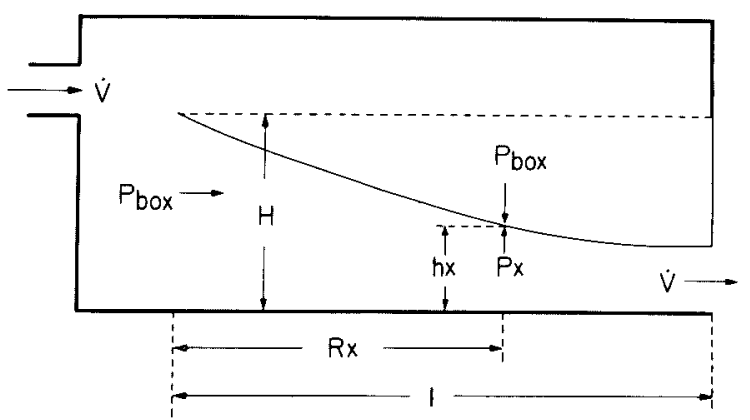

Fig. 4. Mathematical model of the two-dimensional flow channel. For explanation, see text.

sible materials. Driving pressure at the inlet of the channel is $P_{b o x}$ which also can compress the roof to the bottom. $P_{b a_{x}}$ produces flow $(\dot{V})$ in parallel bands and intraluminal pressure $P_{x}$ at distance $x$ from the inlet will be a function of both $x$ and resistance in the airway up to that distance $x$.

(a) Compliance of the elastic bands: To fit the actual relationship between pressure difference $P_{b o x}-P_{x}$ and displacement of the band to the bottom, compliance $C$ of the upper elastic band is simulated as follows; 


$$
C=\frac{\left(H-h_{x}\right)^{a}}{P_{b o x}-P_{x}}
$$

In connection with the definition of complicance, it is necessary to define several points. First, it is assumed that displacement of the upper elastic band is not influenced by its longitudinal stretch. Furthermore, the membrane can move independently; in other words, displacement in the depth at the given segment does not influence displacement at other segments. Finally, at the outlet of airway where displacement of the upper band is apt to be intensified, a stretch of the band in direction of height is disregarded to simplify mathematical treatment, impling $h_{x}=l=H-\left(P_{b o x} C\right)^{1 / a}$, where $h_{x}=l$ is height of the upper band at the outlet.

Fig. 5 shows pressure-displacement relationships of three states of elastic band which is used in the flow model in Fig. 1. The collapsible band was made up by condome latex rubber $9 \mathrm{~cm}$ in width, $27.5 \mathrm{~cm}$ in length and $0.06 \mathrm{~mm}$ in thickness,

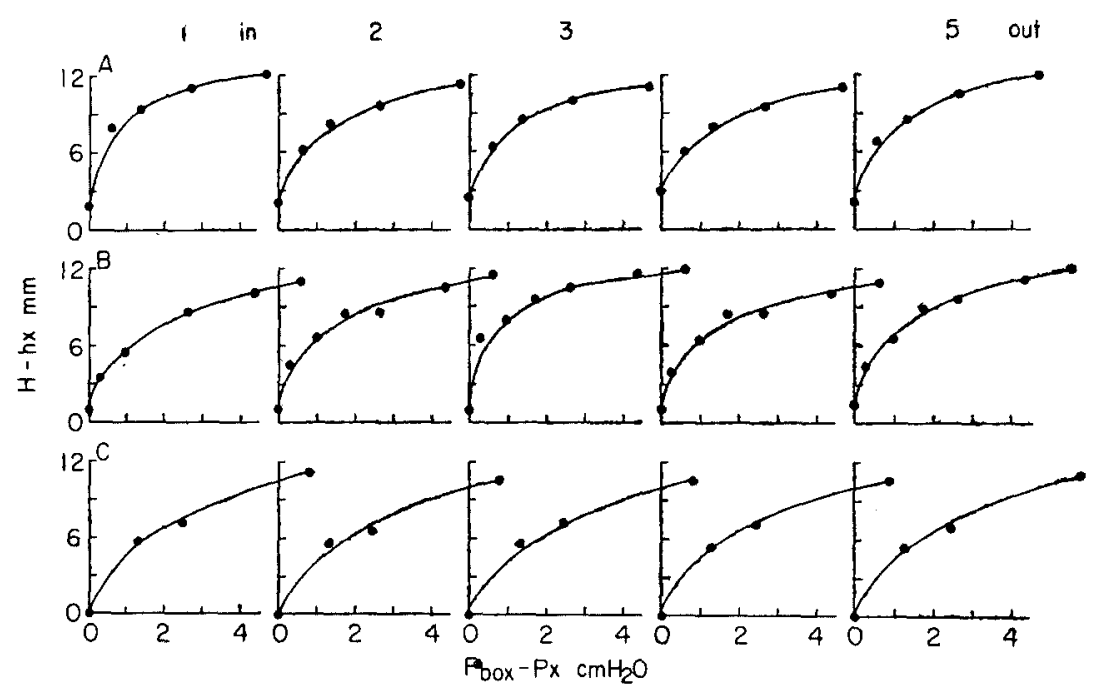

Fig. 5. Static pressure-displacement relationships of three states of the elastic roof used in the flow-model of Fig. 1.

and was divided into five parts by thin rigid bars. In Fig. 5 one can observe that five segments of each membrane have different kinds of pressure-displacement relationship obtained by expanding the membrane in direction of length. Displacements of the membrane were measured at the middle of each segment which usually showed the greatest movement toward the bottom. Among them the membrane A showed the most compliant characteristics and the $\mathrm{C}$ the least ones.

In Fig. 5, pressure-displacement relation of the outermost segment of the membrane A was simulated roughly by the 3.5 powers equation as follows: 


$$
C=\frac{\left(H-h_{x}\right)^{3.5}}{P_{b o x}-P_{x}}
$$

and in the membrane $C$ by the second powers equation.

(b) Relationship between height $h_{x}$ and distance $x$ : Airway resistance $R_{x}$ between $\Delta x$ at distance $x$ may be expressed as follow;

$$
R x=\frac{k \Delta x}{h_{x}^{3}}
$$

where $k$ is a constant which includes viscosity coefficient of gas and other geometrical factors and $h_{x}$ the height at distance $x$. The sum of resistance $R_{0_{-x}}$ along the airway between distance 0 and $x$ is given by the integral of equation (8) with respect to $x$,

$$
R_{0-x}=k \int_{0}^{x} \frac{1}{h_{x}{ }^{3}} d x
$$

By the definitions, the following equation can be derived.

$$
\begin{gathered}
P_{b o x}-P_{x}=R_{0-x} \cdot \dot{V} \\
R_{T}=k \int_{0}^{l} \frac{1}{h_{x}^{3}} d x \\
P_{b o x}=R_{T} \cdot \dot{V}
\end{gathered}
$$

For compliance pressure-displacement relation of the outermost segment of the membrane $A$ is used as follows:

$$
\begin{gathered}
C=\frac{\left(H-h_{x}\right)^{3.5}}{P_{b o x}-P_{x}} \\
\left(H \geq h_{x} \geq 0\right)
\end{gathered}
$$

From eq. (10), (13)

$$
R_{o-x}=\frac{\left(H-h_{x}\right)^{3.5}}{C \dot{V}}
$$

Substituting eq. (14) for eq. (9), one obtains

$$
\frac{\left(H-h_{x}\right)^{3.5}}{C \dot{V}}=k \int_{0}^{x} \frac{1}{h_{x}{ }^{3}} d x
$$

By the operation of intergration, one can obtain

$$
\left(H-h_{x}\right)^{3.5}\left(16 H^{3}+56 H^{2} h_{x}+126 H h_{x}{ }^{2}+231 h_{x}{ }^{3}\right)=429 k C \dot{V} x
$$

This indicates relatinships between the distance $x$ from inlet and the height $h_{\mathrm{x}}$ of upper band. 


\section{3) Behaviors in the segmented collapsible membrane}

Flow model, of which diagram appears in Fig. 1, was constructed to test the validity of eq. (16). With pneumotachometer connected with the box at its inlet, the flow was measured which has produced with the vacuum pump attached to the outlet of the airway. Applied pressure to the airway was measured as pressure difference between box and the outlet of airway. Photoconductive cells at one side of the box were used to convert mechanical movement of the membrane into electrical signal, provided with slitted light source at the other side of collapsible bands. Fig. 7 shows displacement of the membrane at its 5 segments of the airway which were recorded by six-channel pen recorder at the same time which were put together at their corresponding parts of the airway, respectively. Applied pressure was roughly $2.0 \mathrm{~cm} \mathrm{H}_{2} \mathrm{O}$. Each graph is a time plot showing what happened as the applied pressure was raised from 0 to $2 \mathrm{~cm} \mathrm{H}_{2} \mathrm{O}$ and then returned to 0 . The method allows us to pick up displacement of the segment at its middle portion.

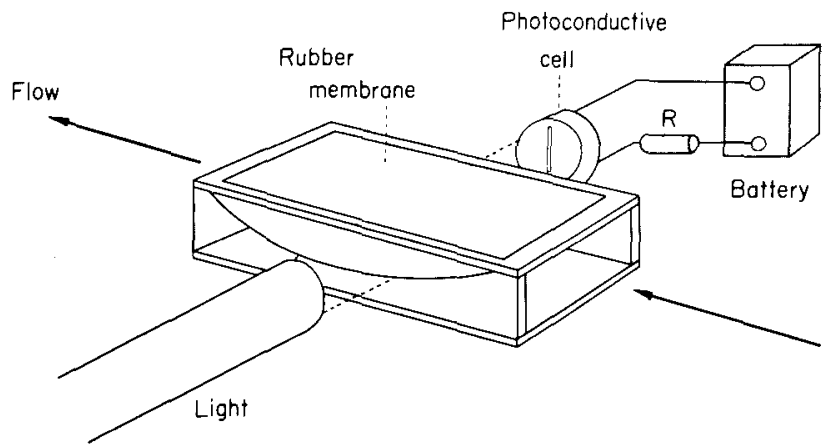

Fig. 6. Photometric apparatus for recording displacement of the membrane.

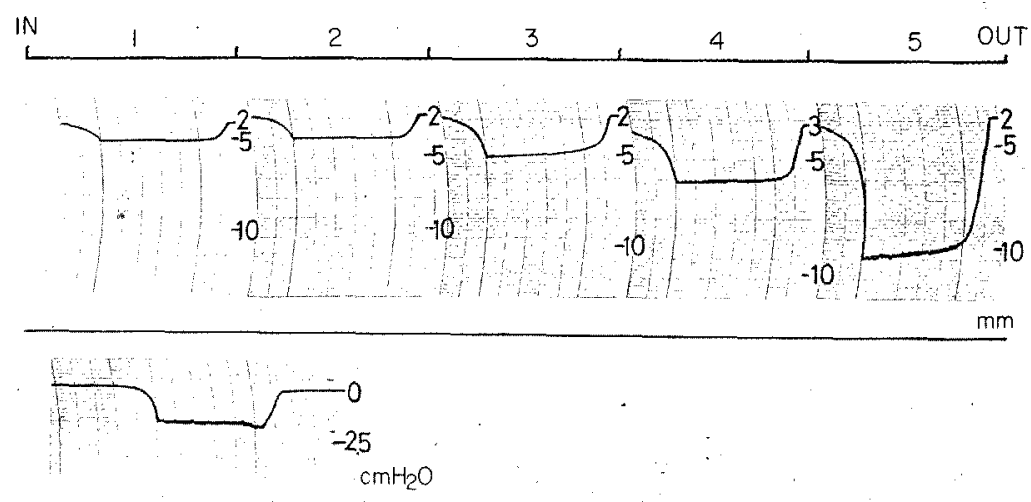

Fig. 7. Displacement of the membrane at its 5 segments of the airway. Each graph is a time plot showing what happened as the applied pressure was raised from 0 to $2 \mathrm{~cm}$ $\mathrm{H}_{2} \mathrm{O}$ and then returned to $\mathrm{O}$. 


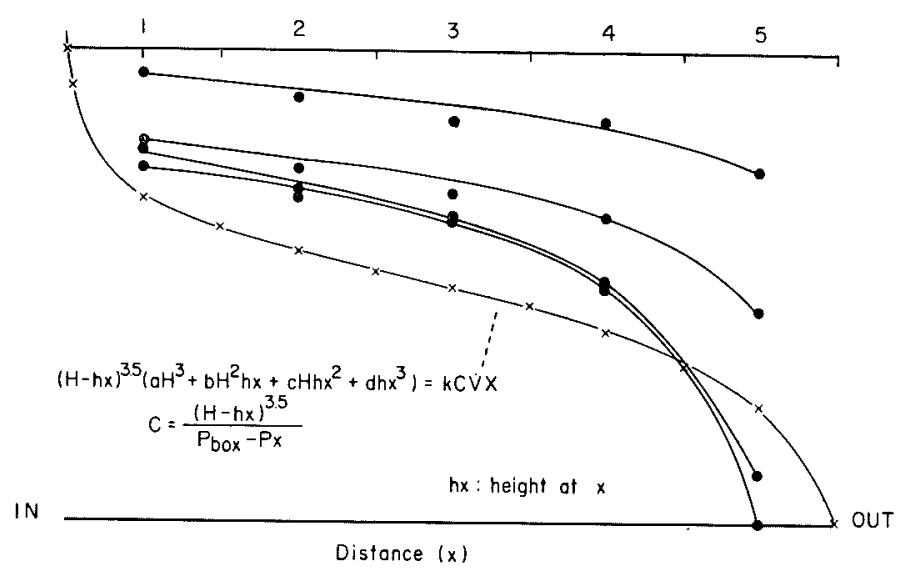

Fig. 8. Displacement of the membrane $\mathrm{A}$ at five segments when four grades of different pressure were applied.

Theoretical $\mathrm{h}_{x}-\mathrm{x}$ relation in the condition 4 of the Table is figured for comparison. a, $\mathrm{b}, \mathrm{c}$ and $\mathrm{d}$ in the equation correspond to $16 / 429,56 / 429,126 / 429$ and 231/429, respectively.

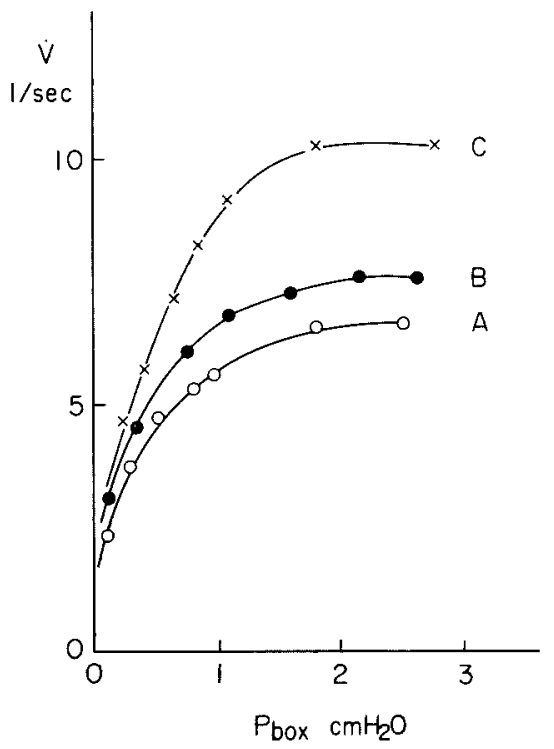

Fig. 9. Pressure-flow curves of the airway with three different states of the membranes $\mathrm{A}, \mathrm{B}$ and $\mathrm{C}$.

Fig. 8 demonstrates displacement of the membrane at five segments at different pressures applied. On the same figure, the theoretical line identical with the line 4 of Fig. 11 is shown for comparison. One can observe considerable similarity between observed displacement and theoretical one. The shift of the experimental curve to the theoretical one is not small, the deformed membrane along the airway 
is quite similar in shape. In Fig. 8 one can observe "expiratory check valve" to occur just at a very limited segment near the outlet of airway. Once the top of fifth membrance nearly approaches to the bottom, the flow reaches at its maximum level with some kind of vibration around the outlet. The frequency of vibration appeared to be roughly in order of some 200 CPS. Next, pressure-flow relationships of the airway were obtained with different compliances of the membranes $\mathrm{A}, \mathrm{B}$ and $\mathrm{C}$ in Fig. 5. Relationship betwen distance $x$ and height $h_{x}$ did not change so much according to the state of the membrane used, but the pressure-flow curve showed definite sift; the lower the compliance was, the higher was the maximum flow, obtained as shown in Fig. 9 In addition, it was observed that a critical pressure difference at which flow was maximum did not show any difference as compliance decreased from $\mathrm{A}$ to $\mathrm{C}$.

\section{Discussion}

High collapsibility of the airway possibly means both increase of compliance of the bronchi and decrease of support of the lung parenchyma surrounding the bronchi. Since compliance of the latex roof of the airway is defined so as to be analogous with the collapsibility in the present paper, the results obtained here may imply that collapsibility of the airway has a significant role to achieve the maximal flow.

Lemoine and Garix (1953) and Wyss (1961) described clinically the bizarre pattern of expiratory narrowing seen in the bronchi of patients with severe airflow limitation and named it "dyskinésie trachéobronchique". The same phenomenon was reported as the expiratory check valve by Dayman (1961, 1951), and Fry (1958) analyzed pressure-flow relationship of the collapsible tubing with a very elegant mathematical tool.

Mead et al. (1967) have considered the lung to be analogous to a Starling resistor during forced expiration, and referred some locus in the airway to EPP, where the lateral intraluminal pressure equals pleural pressure when it is over zero on forced expiration. According to his concept, the EPP moves upstream toward the alveolus with increasing expiratory efforts, and when expiratory flow at a particular lung volume ceases to increase with further increase in pressure, one can assume its position to be fixed, and the maximal expiratory flow $\left(\dot{\mathrm{V}}_{\max }\right)$ may be expressed as follows; $\dot{V}_{\max }=\mathrm{P}_{\mathrm{el}} / \mathrm{R}_{\mathrm{us}}$, where $\mathrm{R}_{\mathrm{u} \text { s }}$ is the resistance of this upstream segment of airway and $\mathrm{P}_{\mathrm{el}}$ is elastic recoil at given lung volume. However, they made no attempt to explain the exact mechanism by which the flow was limited.

Pride and co-workers (1967) have suggested that airway obstruction may be "irreversible" if caused by increased airway resistance from the alveolus to the point at which a waterfall develops, or it may be "reversible" if caused by increased back pressure from bronchomotor tone. The equation of Pride et al. includes not only elastic recoil pressure but also transmural pressure of the collapsible segment which might provide information about the collapse properties of the airways. 
In addition, from correlating careful clinical assessement with pulmonary function test during life and in the excised lung after death, and with study of bronchial and parenchymal pathology, it is predicted that in addition to a loss of radial support from the peribronchial tissue, destruction of the bronchial wall itself which is usually a striking finding in many emphysematous lungs may limit the expiratory flow significantly (Maisel et al. 1968).

Even with the mathematical approach mentioned above, however, many problems remain to be interpreted. That is; (1) To what extent does collapsibility of bronchi limit expiratory flow? (2) How does the EPP cease to move downstream after expiratory check valve completed? It is not an unusual finding that bronchial collapse occurs at a site restricted to the much limited semgent of the airway; that is the segmental bronchus (Macklem et al. 1963, 1965). (3) And finally, what kind of parenchymal support takes place in resisting bronchi to collapse, lung tension only or parenchymal distortion?

From our analysis in the present model, it is clearly demonstrated that collapsibility of the airway directly influences the level of the maximal flow; the less the compliance is the greater the flow occurs. In the lungs, the factors which can resist the collapse of airway are both 'parenchymal radial traction and rigidity of the bronchial wall. In the dog experiment (Takishima et al. 1971) which was carried out by us recently, we succeeded in separating the bronchi from the peripheral alveoli by inserting beads into the bronchi and to measure bronchial collapsibility. The results showed that the bronchi became markedly less compliant and hard to collapse when their volume decreased according to not only decreased compliance of the bronchial wall itself, but also to stress density effect (Mead et al. 1970) of the surrounding lung tissue. Therefore, we consider it to be quite reasonable that collapsible membranes of our model have non-linear characteristics in pressure-displacement relationship as shown in equation 7 , and it may play an important role in order to stabilize the mechanical system. Pride et al. have considered that if the waterfall occurs in the intrapulmonary airway, their Ptm' cannot be a constant independent of lung volume. It is not surprising to say, however, that Ptm' is not a constant but a function of dimension of the airway, wherever the waterfall occurs in extrapulmonary or intrapulmonary airway.

To examine an influence of compliance of the membrane upon the maximal flow through the system, it is needed to obtain pressure-flow relation from equations 16,11 and 12 . However, equation 16 is so complicated that it is not possible to solve. Accordingly, two conditions in compliance of the membrane were chosen to obtain pressure-flow relationships in the flow system as follows;

Condition I:

$$
C_{1}=\frac{H-h_{x}}{P_{0}-P_{x}}
$$

Condition II :

$$
C_{2}=-\frac{\ln H-\ln h_{x}}{P_{0}-P_{x}}
$$

In the condition I, displacement of the membrane has a linear relationship to 
transmural pressure, and the height $h_{\mathrm{x}}$ will be zero in a limited magnitude of transmural pressure, $P_{\mathrm{o}}-P_{x}=H / C$. On the contrary, in the condition II, transmural pressure has an exponential function of the height $h_{x} ; h_{x}=H \cdot e^{-C\left(P_{0}-P_{x}\right)}$, and infinite pressure will be necessary to compress the membrane completely to the the bottom; $h_{x}=0$. The relationships between the distance $x$ and the height $h_{x}$, pressure-flow

TABLE 1. The $h_{x}-x$-relation, pressure-flow relation, maximum flow rate $\left(\dot{V}_{\text {max }}\right)$ and the driving pressure at $\dot{V}_{\max }\left(P_{b o x}\right.$ at $\left.\dot{V}_{m x x}\right)$ calculated in the mathematical model with 4 kinds of compliance

\begin{tabular}{|c|c|c|c|c|c|}
\hline & Compliance $C$ & $h_{x}-x$ relation & $P-\dot{V}$ relation & $\dot{V}_{\max }$ & $\begin{array}{l}P_{b o x} \text { at } \\
\dot{V}_{\text {max }}\end{array}$ \\
\hline 1 & $\frac{H-h_{x}}{P_{b_{o x}}-P_{x}}$ & $h_{x}^{4}=H^{4}-4 K C \dot{V} x$ & $\dot{\mathrm{V}}=\frac{H^{4}-\left(H-P_{b o x} C\right)^{4}}{4 K C l}$ & $\frac{H}{4 C R}$ & $\frac{H}{C}$ \\
\hline 2 & $\frac{\ln H-\ln h_{x}}{P_{b_{0 x}}-P_{x}}$ & $h_{x}^{3}=H^{3}-3 K C \dot{V} x$ & $\dot{\mathrm{V}}=\frac{H^{3}}{3 K C l}\left(\mathbf{1}-e^{-3} C P_{b o x}\right)$ & $\frac{1}{3 C R}$ & $\infty$ \\
\hline 3 & $\frac{\left(H-h_{x}\right)^{2}}{P_{b_{0} x}-P_{x}}$ & $\begin{array}{l}h_{x}^{4}\left(4 h_{x}-5 H\right)+H^{5} \\
=10 K C \dot{V}_{x}\end{array}$ & $?$ & $?$ & $?$ \\
\hline 4 & $\frac{\left(H-h_{x}\right)^{3,5}}{P_{b o x}-P_{x}}$ & $\begin{array}{l}\left(H-h_{x}\right)^{3.5}\left(16 H^{3}\right. \\
\quad+56 H^{2} h_{x}+126 H h_{x}^{2} \\
\left.\quad+231 h_{x}^{3}\right)=429 K C V x\end{array}$ & $?$ & $?$ & $?$ \\
\hline & & $R=\frac{k l}{H^{3}}$, & $k=-\frac{12 \eta}{m}$ & & \\
\hline
\end{tabular}

$R$ is airway resistance of the parallel channel under uncompressed state, and $k$ a constant.

relationships, the maximal flow and the pressure at the maximal flow in these two different conditions are listed in Table I. The maximal flow is obtained by differentiating the flow by the pressure, being the flow at $d \dot{V} / d p=0$, in the equation of pressure-flow relationships shown in the Table.

It is quite interesting to see that in both conditions the maximal flow has an inverse relation with compliance of the membrane. Although, in the condition I it is not posible to see what happens when the pressure $P_{0}$ increases beyond a critical pressure, $H / C$, because the equation described above can be applied only to the condition of $H / C \geq P_{0} \geq 0$, there might supposedly appear some unstable condition such as vibration of the membrane.

In the condition II, however, the pressure-flow of the system shows some exponential relation and the flow never can reach its maximum. In the lung, the transmural pressure of the membrane at the maximum flow corresponds to Ptm' in the equation (2) by Pride et al. (1967). Today, we have no information to decide which condition can be applied to the real tracheobronchial system. According to our finding (Takishima et al. 1971; Fig. 10), however, the bronchi become markedly less compliant and hard to collapse when the bronchi are decreasing their volume, and the radius of the bronchi estimated from their volume change is roughly correlated linearly with transmural pressure between the alveolar space and 

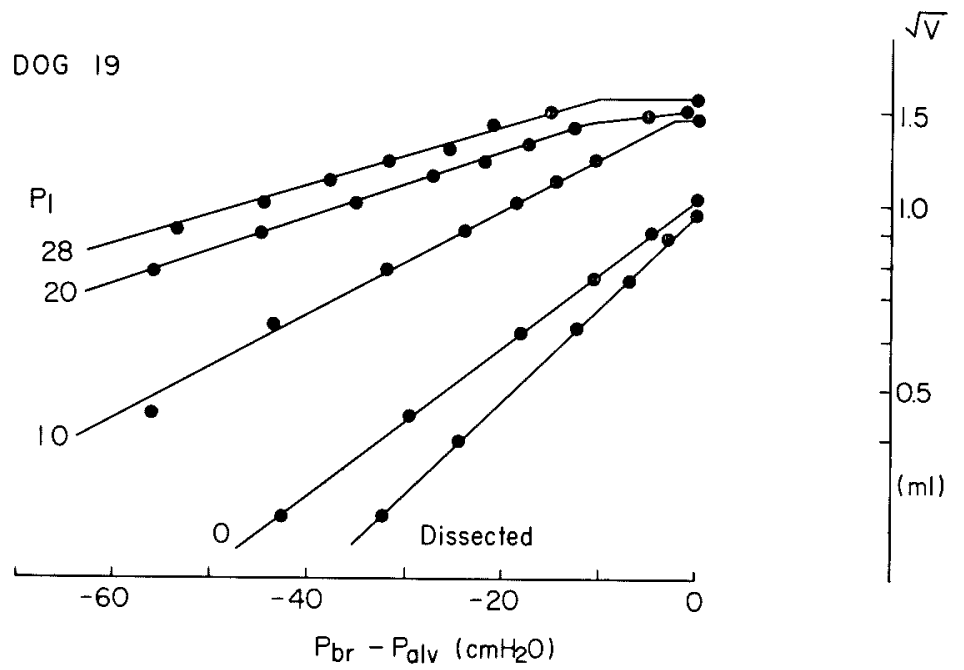

Fig. 10. Pressure-radius curves of the contral bronchi separated from peripheral bronchi with beads in the dog lung. Pressure is transmural pressure between alveolar pressure $\left(\mathrm{P}_{\mathrm{al}}\right)$ and intrabronchial pressure $\left(\mathrm{P}_{\mathrm{br}}\right)$. To estimate the radius change of the bronchus from its volume change, the volume is expressed by its square root by assuming the length to be constant.

the bronchial lumen when the radius is expressed on a logarithmic scale. In this case, the condition II is most probable at least in the normal lung, and it is not possible to find a limited value of Ptm' (Pride et al. 1967).

Hitherto, it has been strongly suggested that the locus of waterfall must be located between the segmental bronchi and the trachea in the normal subject (Macklem et al. 1963, 1965). In the present experiment, it was clearly demonstrated that if the airway could be assumed as a uniform elastic tube, the locus of waterfall would be at the uppermost portion of the airway. It is interesting that the degree of collapse appeared to be much intense at the outermost portion, suggesting that the locus of waterfall would be fixed at very narrow segment of the airway. In the lung, however, different portions of the bronchi might not have the same degree of collapsibility (Martin et al. 1958, Croteau et al. 1961, Staub 1963, Olsen et al. 1967). In this case, it might be suggested that the uppermost portion of the more compliant segment would be the locus of waterfall, which would correspond to the portion suggested by Macklem and Wilson (1965).

In Fig. 11, relationships between the distance $x$ and the displacement $h_{x}$ are summarized, which are calculated in the system with 4 kinds of compliance of the elastic membrane. Curves in the figure are constructed so as to be $H=1$ and $l=1$ in the model (Fig. 4); $h_{x}=1$ at $x=0$ and $h_{x}=0$ at $x=1$. The condition $h_{x}=0$ at $x=1$ means that the maximal flow is established in the conditions I and II of the Table. In the conditions III and IV, however, there is no information on whether or not the maximal flow is established, although it is strongly suggested. 


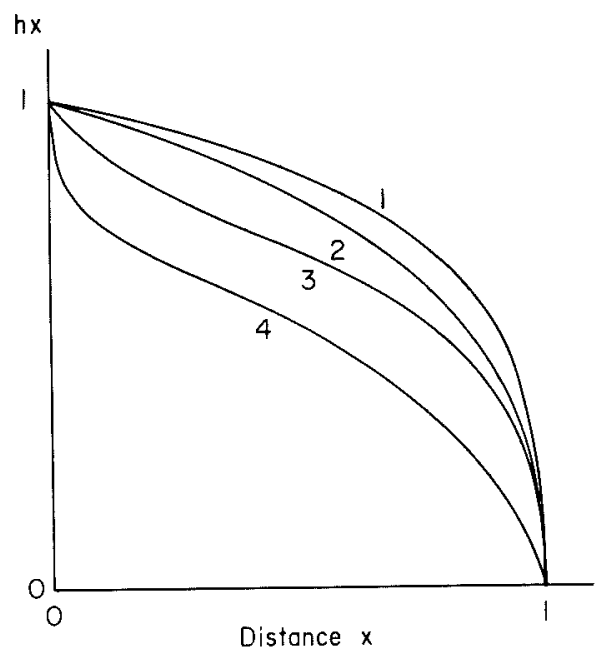

Fig. 11. Relationships between distance $x$ and height $h_{x}$ calculated in the mathematical model with 4 kinds of condition. Numbers correspond to those in the Table. For explanation, see text.

From the equations which define the maximal flow and $h_{x}-x$ relationships shown in the Table, $h_{x}-x$ relation at the maximal flow can be derived in the conditions I and II as follows;

Condition I:

$$
h_{x}^{4}=H^{4}-\frac{H^{4}}{l} x
$$

Condition II:

$$
h_{x}^{3}=H^{3}-\frac{H^{3}}{l} x
$$

As seen in equations 19 and 20, compliances, $C$, are dropped from the equations, indicating that compliance itself does not influence $h_{x}-x$ relationship when the maximum flow is reached, and what does really change its relation is the powers of $\left(H-h_{x}\right)$.

Thus, increase of the powers of $\left(H-h_{x}\right)$ in the eq. (6), which means increased collapsibility of the membrane, has a marked effect of compressing the innermost segment of the membrane to the bottom. This effect was confirmed by the experiment with a different kind of the membrane

On the contrary, if compliance of the membrane is much less collapsible, segments upstream to the portion of waterfall are hard to be compressed and the outermost portion collapses abruptly only at a very limited segment in length. In the lung, therefore, it is suggested that the bronchi can collaspe abruptly, the less compliant the more restricted in length. However, it must be mentioned that collapsibility of the membrane does not change the phenomenon that the membrane as a whole is convex in shape to the roof of the bands and the outermost segment of the flow system chiefly limits the flow. 
In Fig. 9 it was demonstrated that the maximal flows were establisched at a fixed driving pressure of about $2 \mathrm{cmH}_{2} \mathrm{O}$, regardless of collapsibility of the memrane. From the relation between transmural pressure and displacement of the collapsible membrane obtained by static measurement (Fig. 5), it was simply deduced that there still remained a considerable amount of the gap between the two bands. The height $h_{x}$ of the membrane at transmural pressure of $2 \mathrm{cmH}_{2} \mathrm{O}$ was roughly $2.5 \mathrm{~mm}$ at the $A, 3 \mathrm{~mm}$ at the $\mathrm{B}$ and $5.5 \mathrm{~mm}$ at the $\mathrm{C}$ membrane, respectively. If we consider that the maximal flow can be reached as the gap $h_{x}$ between the two bands approaches to zero, then it is suggested that the transmural pressure enough to make complete collapse of the membrane $\left(h_{x}=0\right)$ must exist.

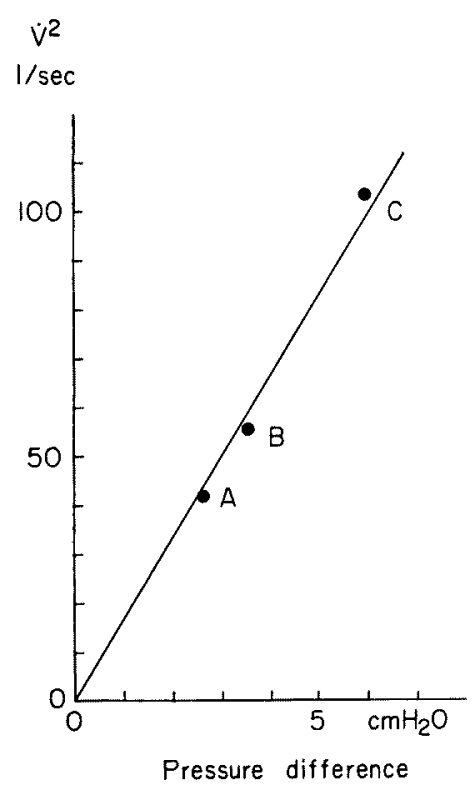

Fig. 12. Maximum flow rate squared and pressure excess required to achieve complete collapse. A, B and C mean three different states of the membrane. For explanation, see text.

In Fig. 5, the excess of pressure necessary to produce complete collapse of the membrane was $2.7 \mathrm{~cm} \mathrm{H}_{2} \mathrm{O}$ at the $\mathrm{A}, 3.6 \mathrm{~cm} \mathrm{H}_{2} \mathrm{O}$ at the $\mathrm{B}$, and $6.0 \mathrm{~cm} \mathrm{H}_{2} \mathrm{O}$ at the $\mathrm{C}$ membrane at their uppermost segments, respectively. A number of complicated explanations are possible to account for this pressure excess. However, Fig. 12 shows a good correlation between the pressure excess and the maximal flow squared, suggesting that the excess of pressure can be at least partly understood by the Bernoulle's effect.

\section{SUMMARY}

We made a two-dimensional flow model composed of two bands in parallel. The roof was made with the collapsible condome rubber and segmented into 
five parts by the rigid ribs to keep interdependence in movement of each segment and the bottom with the plastic plate. The flow can occur between the bands when it is positioned in the box which can generate a driving pressure, and the driving pressure in the box can also compress the elastic roof toward the bottom, thus resulting in an increase of airway resistance, that is, "expiratory check valve." The results obtained are as follows: First, pressure-flow relationships of the airway demonstrated the flow to reach its maximum level at a certain pressure, and the lower the compliance of the elastic roof was, the higher the maximum flow rate was. Next, it was observed that displacement of the collapsible roof toward the bottom occurred just at a very limited segment near the outlet of airway, showing the membrane as a whole to be convex in shape to the roof of the bands, and the flow was limited chiefly by the outermost segment of the airway. Finally, it was proved that the present results roughly coincided with the theoretical calculation based on the mathematical two-dimensional model. Our recent experiments on dogs revealed that the bronchi surrounded by the lung parenchyma became markedly less collapsible as the radius decreased. From the above theoretical analysis and our experimental results, it was concluded that the maximum flow had an inverse relation with both compliance and resistance of the bronchi. Furthermore, displacement of the collapsible roof toward the bottom appeared at a very limited segment near the outlet of airway. This means that the stress on the membrane focuses into much narrower segment and "expiratory check valve" occurs at a very limited segment of the bronchi.

\section{Acknowledgment}

We acknowledge the kind help of Mr. T. Sakurai and thank Dr. J. Mead for his discussion.

A preliminary report was presented at the satellite symposium "Models in ventilatory mechanics" of 25th International Congress of Physiological Sciences, held in Paris, 1971.

This study was supported in part by the Grant from the Ministry of Education.

\section{References}

1) Croteau, J.R. \& Cook, C.D. (1961) Volume-pressure and length-tension measurements in human tracheal and bronchial segments. $J$. appl. Physiol., 16, 170-172.

2) Dayman, H. (1961) The expiratory spirogram. Amer. Rev. resp. Dis., 83, 842-855.

3) Dayman, H. (1951) Mechanics of airflow in health and in emphysema. J. clin. Invest., 30, $1175-1190$.

4) Fry, D.L. (1958) Theoretical considerations of the bronchial pressure-flow-volume relationships with particular reference to the maximum expiratory flow volume curve. Phys. Med. Biol., 3, 174-194.

5) Lemoine, J.M. \& Garaix, J.P. (1953) Leo dyskinésies trachéo-bronchiques à forme hypotonique. Sem. Hôp. Paris, 29, 933-942.

6) Macklem, P.T., Fraser, R.G. \& Bates, D.V. (1963) Bronchial pressures and dimensions in health and obstructive airway disease. J. appl, Physiol., 18, 699-706.

7) Macklem, P.T. \& Wilson, N.J. (1965) Measurement of intrabronchial pressure in man. J. appl. Physiol., 20, 653-663. 
8) Maisel, J.C., Silvers, W., Mitchell, R.S. \& Petty, T.L. (1968). Bronchial atrophy and dynamic expiratory collapse. Amer. Rev. resp. Dis., 98, 988-997.

9) Martin, H.B. \& Proctor, D.F. (1958) Pressure-volume measurements on dog bronchi. J. appl. Physiol., 13, 337-343.

10) Mead, J., Takishima, T. \& Leith, D. (1970). Stress distribution in lungs: a model of pulmonary elasticity. J. appl. Physiol., 28, 596-608.

11) Mead, J., Turner, J.M., Macklem, P.T. \& Little, J.B. (1967) Significance of the relationship between lung recoil and maximum expiratory flow. J. appl. Physiol., 22, 95-108.

12) Olsen, C.R., Stevens, A.E. \& McIlroy, M.B. (1967) Rigidity of tracheae and bronchi during museular constriction. $J$. appl. Physiol., 23, 27-34.

13) Pride, N.B., Permutt, S., Riley, R.L. \& Bromberger-Barnea, B. (1967) Determinants of maximal expiratory flow from the lungs. J. appl. Physiol., 23, 646-662.

14) Staub, N.C. (1963) The interdependence of pulmonary structure and function. Anesthesiology, 24, 831-854.

15) Takishima, T. Sasaki, H. Sasaki, T. \& Nakamura, T. (1971) Influence of lung parenchyma on collapsibility of dog bronchi. Proceedings of the International Union of Physiological Sciences, 9, 556.

16) Wyss, F. (1961) Dyskinésie trachéo-bronchique. Bronches, 11, 11-25. 